\title{
ONTOLOGY ASSISTED MODELLING OF GALVANIC CORROSION OF MAGNESIUM
}

\section{DANIEL HOECHE ${ }^{1}$, NATALIA KONCHAKOVA ${ }^{1}$, MIKHAIL L. ZHELUDKEVICH ${ }^{1 ; 2}$, THOMAS F. HAGELIEN ${ }^{3}$ AND JESPER FRIIS ${ }^{4}$}

${ }^{1}$ Institute of Surface Science, Helmholtz-Zentrum Geesthacht, Max-Planck Str. 1, Geesthacht, 21502, Germany, daniel.hoeche@hzg.de, natalia.konchakova@hzg.de, mikhail.zheludkevich@hzg.de

${ }^{2}$ Faculty of Engineering, Kiel University, Kaiserstrasse 2, 24143 Kiel, Germany

${ }^{3}$ SINTEF-Ocean, NO-7465 Trondheim, Norway, thomas.f.hagelien@sintef.no,

${ }^{4}$ SINTEF-Industry, NO-7465 Trondheim, Norway jesper.friis@sintef.no

Key words: Data Inter-Exchange, Metadata Structures, Semantic Interoperability, Multiscale Framework.

\begin{abstract}
Multi-physics and multiscale modelling frameworks, especially for the computer based analysis of surface damage and corrosion, requires not only robust computational tools but also an efficient data-centric architecture for handling information exchange at different modelling scales. The issue to exchange data provided by different computational solvers as well as required and used in different programming languages forms a request in specific formats signifying a strong non-uniformity for an easy nexus with other solvers. This non-uniformity has created a need to focus on intermittent state-of-theart data-centric software tools, which aim to bridge data exchange technology, to ensure heterogeneity across a wide range of solvers [1]. Moreover, data organization in the form of ontological representation and metadata structures are necessary to be prepared as a standard for a coherent representation of information regardless of the diverse nature of data formats specific to a scientific discipline. A domain ontology based on the European Materials \& Modelling Ontology (EMMO) for galvanic corrosion is outlined and connected to corresponding concepts in a galvanic corrosion model. This fundamental work provides and discusses the concept, underlying terminology and working mechanism of a data-centric architecture for exchanging and interfacing data-flow between data sources/sinks and solvers. It realizes an ontological representation of physics and chemistry of galvanic corrosion. Thus, it is a nuclei for further improvement's of interoperability between complex corrosion related phenomena and models. It paves the way for accurate computational corrosion engineering.
\end{abstract}

\section{INTRODUCTION}

Damage and corrosion are huge issues for engineering of multi-material structures and for the construction sector, which have a high economic impact [2]. An accurate service-life prediction and maintenance action are challenging scientific and industrial problems on which researchers and manufacturing specialists are working together. Successful predictions of corrosion and extension of service-life of en- 
gineering structures and lightweight multi materials systems can bring till $8 \%$ of world $\mathrm{CO}_{2}$ emission and contribute sufficiently to the EU Green deal course. Modelling and simulation provide fundamental support in this process. Currently some modelling approaches are developed to analyse corrosion of materials surface, damage state and failure due to the materials degradation. Advanced simulation techniques provide the access to different software and data, which should be available and exchangeable in numerical analysis of the complex non-liner multi-scale modelling problem of corrosion, bridging different simulation tools on different simulation steps and levels [4]. Moreover, the computation challenge includes different issues with homogenization, accuracy, real-time validation and other functional complexities [1]. Unfortunately, exchange of data provided by different computational solvers is very often strong non-uniform due to use of different programming languages and application of different modelling stiles. To solve this software engineering and scientific issue and support an easy nexus between modeling codes and data with different solvers, the standardisation of modelling workflows, in form of materials modelling data table (MODA) description, data organization and providing ontological representation of models and used or generated data are required [3]. Moreover, the ontological based modelling and simulation would provide support CAD, predictive maintenance and contribute to the detail analysis of risk management in multi-functional constructions.

In this paper, the authors would like to demonstrate an ontology based modelling approach applied to a simple example of predictive modelling of galvanic couple of magnesium based components in light weight structural design. The degradation of galvanic coupled materials is a well known problem, especially for constructors. Due to the dissolution of the magnesium alloys in aggressive environments

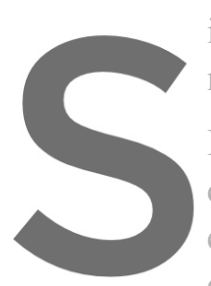
in contact with the more

nice to have a tool to com

In this study, we simulate 1

copper on a very simple ge

electrochemical fundantenton

corrosion model. Both contact partners were chost
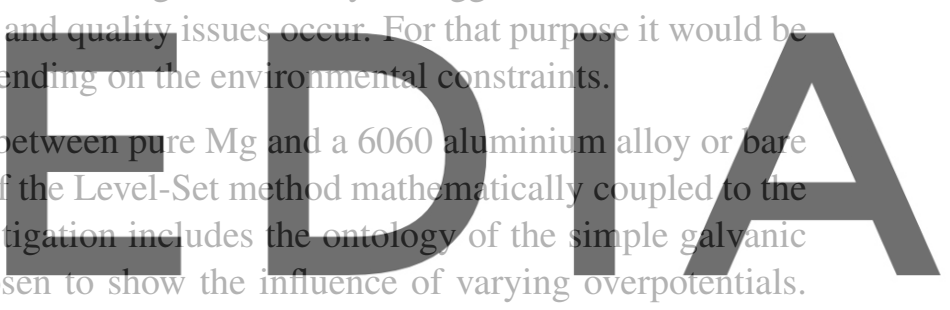

In the literature nice investigations by Desphande [5, 6, 7], Murer [8], Jia [9, 10] or Lacroix [11] can

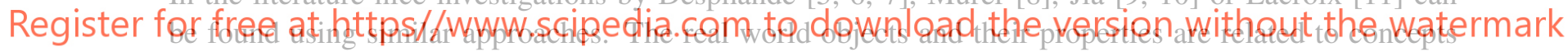

in a domain ontology for galvanic corrosion based on the European Materials \& Modelling Ontology

(EMMO) [12]. This study will give a base for further investigations and offer some possibilities to implement the involved chemical and physical processes on a "volumetric" surface [1].

\section{Ontology for corrosion modelling}

\subsection{Problem description domain: computation of galvanic corrosion}

For the simulation of current distributions, the Laplace equation is solved and coupled to the Level-Set equations according [13]. The electrode kinetics was described by the Buter-Volmer equation and by a modified version according Murer et.al [14]. Figure 1 shows the considered geometry and materials domains.

The fundamental approach is to set the Level-Set variable $\phi$ at the magnesium surface to 0.5 . Boundary numbers and Level-Set initials are indicated in the Figure 1. $\eta=U_{s}-U_{l}-E_{0}$ is the over potential, which depends on the electrode (extern) potential $U_{s}$ (here $U_{s}=0$ ), the electrolyte potential $U_{l}$ and the equilibrium potential $E_{0} . i$ is the current. The boundary conditions (BCs) are presented in Table 1. 


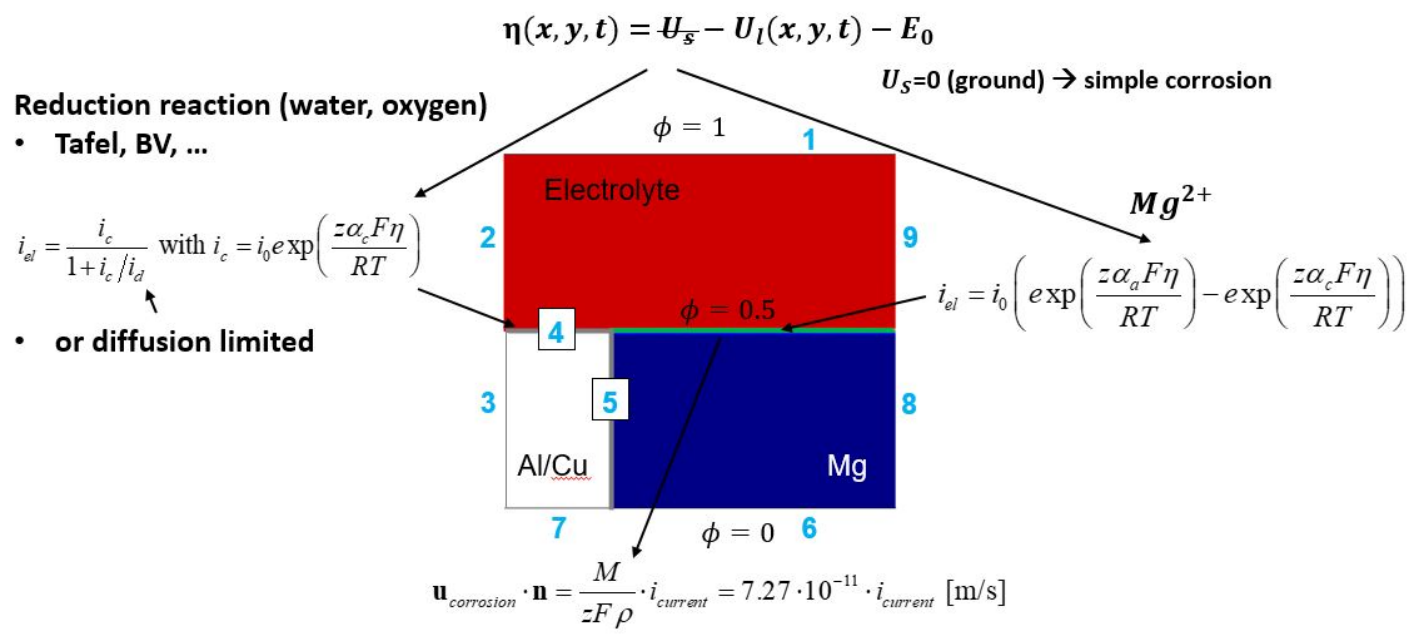

Figure 1: Materials domain, geometry, boundaries and applied materials relations used for simulation. The blue numbers label the different boundaries in the model.

Table 1: Boundary conditions at the considered geometry.

$\begin{array}{ccc}\text { Boundary } & \text { Laplace } & \text { Level-Set } \\ 1 & i=0 & \phi=1\end{array}$
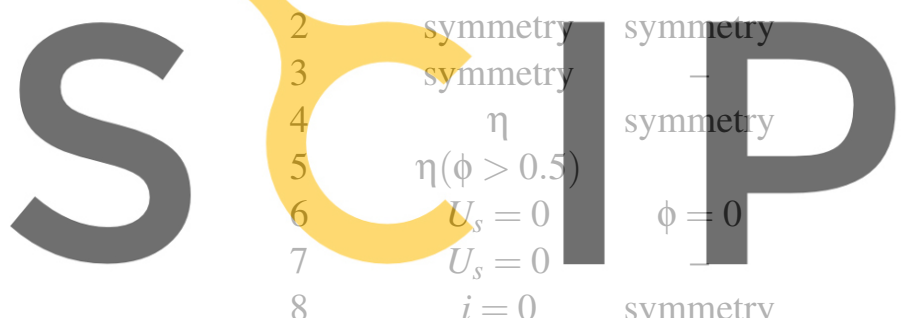

symmetry
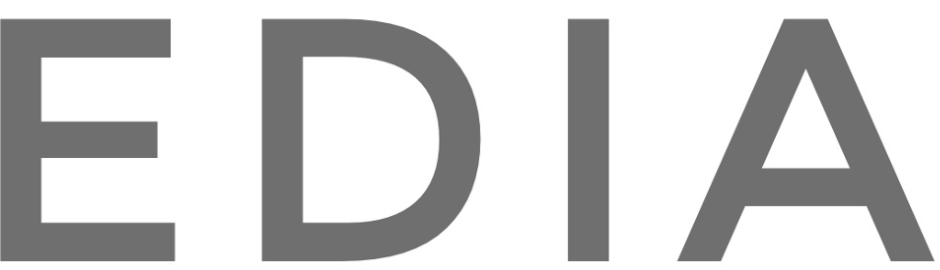

Register for free at https//ww woipedia.com to download the version without the watermark

\subsubsection{Electrochemistry}

The model is based on very simple assumptions by neglecting convection, diffusion and concentration gradients. Thus, the Nernst equation which describes the electrolyte is reduced to the Laplace equation $\Delta U=0$ in the simplified model and the following equations occur:

$$
\nabla \mathbf{j}_{l s}=Q_{l s} \text { and } \mathbf{j}_{l s}=-\sigma \cdot \nabla U_{l, s}
$$

Here $l$ and $s$ indicate the electrolyte respectively the electrode, $\mathbf{j}$ describes the current density vector, $U$ the electrical potentials and $\sigma$ the effective conductivity. $Q$ as a source term becomes zero. At the electrodeelectrolyte interface, the standard Butler-Volmer equation in $\mathrm{Mg}$ and the modified Butler-Volmer equation according Murer et.al [14] in $\mathrm{Al}$ and $\mathrm{Cu}$, were used to explain and describe the kinetics (empirical). The required parameters were taken from the experimental studies and fittings. 


$$
i_{e l}=i_{0}\left[\exp \left(\frac{z \alpha_{a} F \eta}{R T}\right)-\exp \left(\frac{z \alpha_{c} F \eta}{R T}\right)\right]
$$

$z$ is the electron number, $F$ is the Faraday constant, $T$ is room temperature, $R$ is the molar gas constant, $\eta$ is the overpotential, $i_{0}$ is the exchange current, and $\alpha_{a}, \alpha_{c}$ are the anodic and cathodic coefficient, respectively. The modified equation just describes the cathodic branch and looks like:

$$
i_{e l}=\frac{i_{c}}{1+i_{c} / i_{d}} \text { with } i_{c}=i_{0} \exp \left(\frac{z \alpha_{c} F \eta}{R T}\right)
$$

The difference results from $i_{d}$ which describes the current limitation due to diffusion (reduction) processes mainly based on oxygen. This effect was discussed by Hoeche and Isakovic in [20]. The fitting parameters, used for the simulation and ontology, are presented in Table 2.

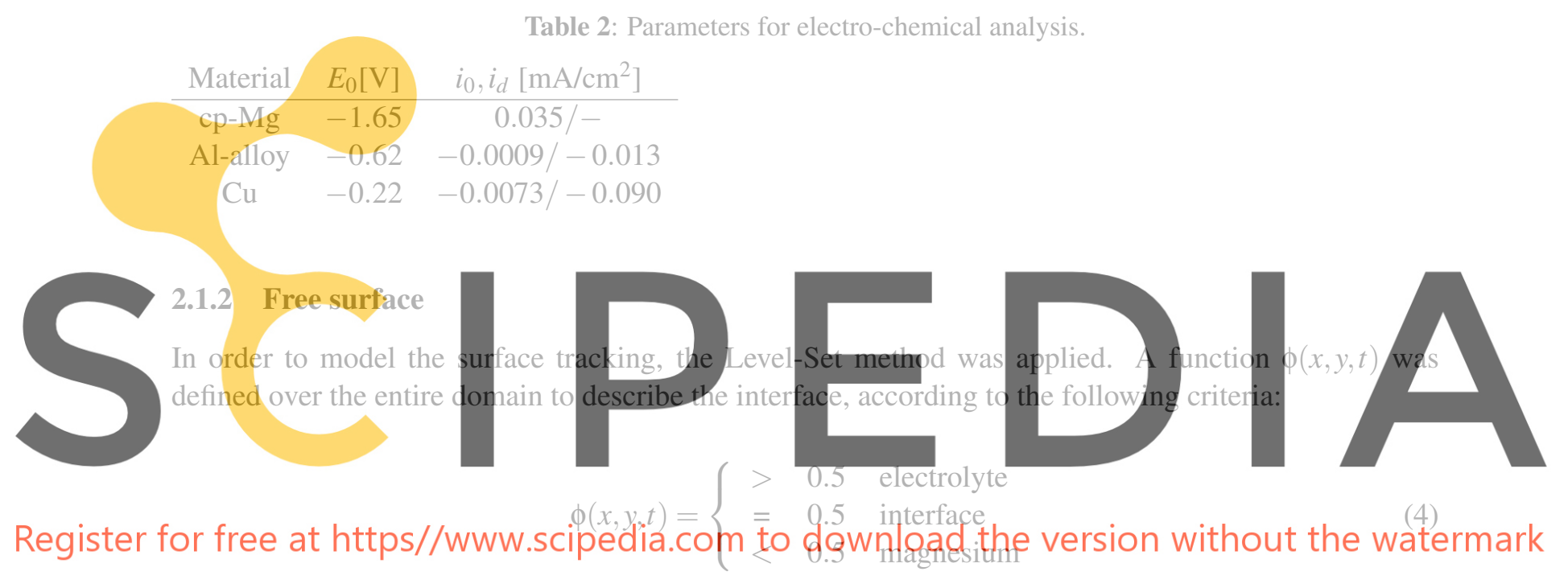

The interface movement can be described by a simple partial differential equation, which can be solved simultaneous numerically with the Laplace equation. Olsson et.al. [15] used the following expression:

$$
\frac{\partial \phi}{\partial t}+\mathbf{u}_{c o r r} \cdot \nabla \phi=\gamma_{L} \nabla \cdot\left(\varepsilon((\nabla \phi \cdot \mathbf{n}) \mathbf{n})-\phi(\mathbf{1}-\phi) \frac{\nabla \phi}{|\nabla \phi|}\right)
$$

Here the vector $\mathbf{u}_{\text {corr }}$ is the speed of the surface tracking, which is considered in this case as the corrosion rate. The speed is a result of the Laplace equation and the normal vectors of the interface $\mathbf{n}$. The interface thickness $\varepsilon$ was set to $\varepsilon=3 h$ (where $h$ is the mesh size). $\gamma_{L}$ is a stabilization parameter that determines the repetition of re-initialization for each time step. This parameter has the unit $[\mathrm{m} / \mathrm{s}]$ and is in the order of the maximum velocity of approximately $10^{-7}-10^{-9}$ appearing from the corrosion rate. For accurate computation tuning becomes necessary. The interface normal vector is defined by $\mathbf{n}=\frac{\nabla \phi}{|\nabla \phi|}$ at $\phi=0.5$ and the surface velocity for $\mathrm{Mg}$ is according to Faraday's law: 


$$
\mathbf{u}_{\text {corr }} \cdot \mathbf{n}=\frac{M}{z F \rho} \cdot i_{\text {current }}=7.27 \cdot 10^{-11} \cdot i_{\text {current }}[\mathrm{m} / \mathrm{s}]
$$

where $M$ is the atomic mass, $z$ is the electron number, $F$ is the Faraday constant and $\rho$ is the density of the corroding Mg. $F=96,485.34 \mathrm{C} \mathrm{mol}^{-1}, M=23.98 \mathrm{~g} \mathrm{~mol}^{-1}, z=2, \rho=1770 \mathrm{~kg} \mathrm{~m}^{-3}$ for the corroding $\alpha$-phase. A smoothed continuously differentiable function was defined to accommodate the boundary conditions at the interface:

$$
\delta=6 \nabla \phi \cdot|\phi(1-\phi)|
$$

Thereby, it is possible to transform boundary phenomena to volumetric ones. Due to the smeared out interface, the material parameters along the interface were described by Eq. (8), whereas $f$ corresponds to the different physical properties (e.g. $\sigma$ ) of the electrolyte and the magnesium.

$$
f=f_{M g}(\phi<0.5)+f_{e l}(\phi>0.5)
$$

The initial level set function $\phi_{0}$ was set according to the model build up in Figure 1.

\subsubsection{Experimental data}

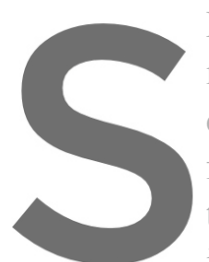

Experimental input dat

minium 6060 and $99.5 \%$

$\mathrm{cm}$ which were joined

foursquare acrylic glass

tively. The assây was

installed which documented the expt
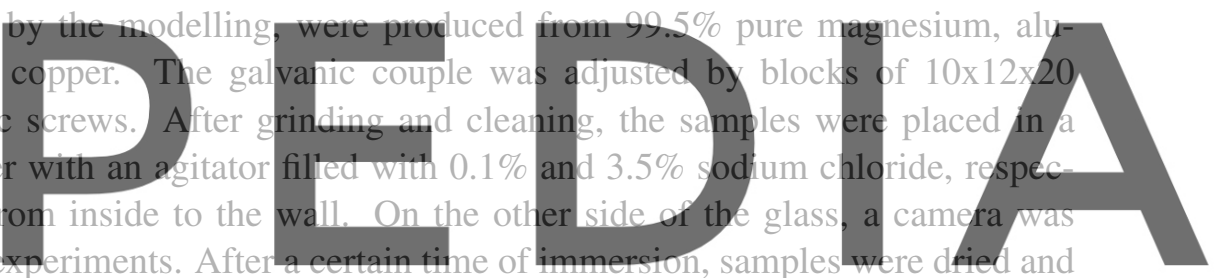

cleaned by chromic acid to remove corrosion products.

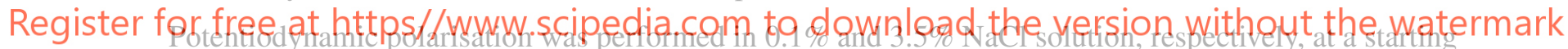

$\mathrm{pH}$ of 6.5. The corrosion cell $(333 \mathrm{ml})$ with a three electrode set-up consisted of an $\mathrm{Ag} / \mathrm{AgCl}$ reference, a

$\mathrm{Pt}$ counter electrode and the specimen as working electrode. The electrolyte temperature was $22 \pm 0.4^{\circ} \mathrm{C}$ and the electrolyte was stirred during the experiments.

\subsection{Ontologising galvanic corrosion}

In order to enable a semantic description of the galvanic corrosion model, we will in this section outline a domain ontology for galvanic corrosion that the real world objects and physical quantities in our corrosion model can be related to.

Figure 2 shows the ontologisation of the three domains and the three internal boundaries in Figure 1 as individuals. Their corresponding material subclasses are also shown. The boundaries are related to the domains via hasContactWith relations (illustrated as double-headed arrows to emphasise that it is symmetric). For simplicity are the external boundaries not included in the figure.

Figure 3 shows how the anode, cathode and electrolyte individuals are related to their corresponding formulation of the Laplace equation for the three domains (da, dc, de) with hasModel relations. These 


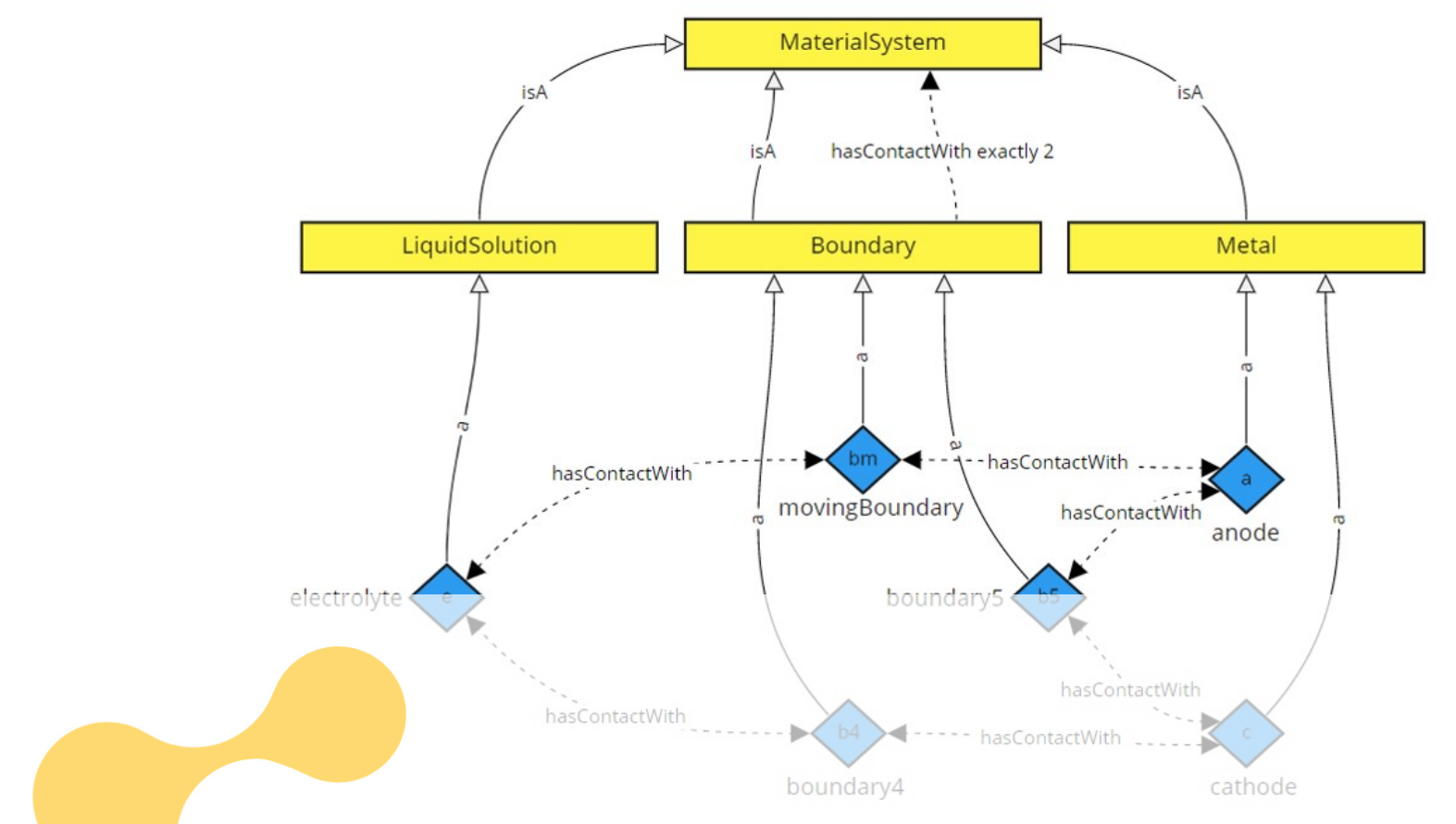

Figure 2: Real world material classes (yellow boxes) and individuals (blue diamonds) considered galvanic corrosion model. The individuals refer to the domains (anode: $\mathrm{Mg}$, cathode: $\mathrm{Cu}$ and electrolyte) and some selected

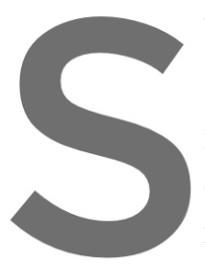

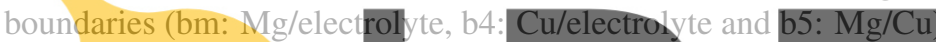

instances of the Laplace equation are related to their

corresponding solutions

relations. Spatial direct pa

(ea, ec, ee)

e), which are ins
is used, because
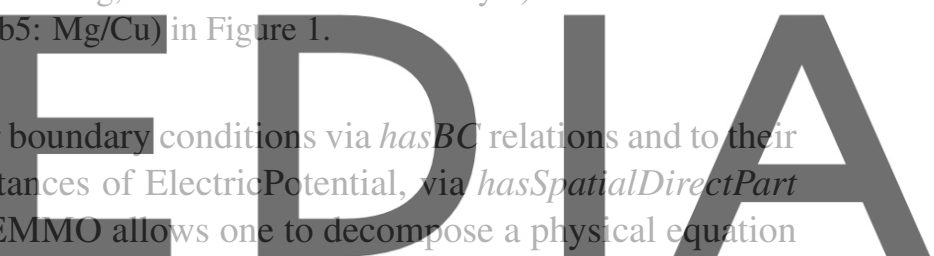

into its spatial direct parts, being operators, numbers and physical quantities.

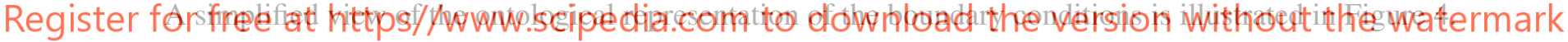
hasModel relations are used to relate the three inner boundaries (b5, b4, bm) to their corresponding boundary conditions (bc5, bc4, bcm). These boundary conditions are material relations and have overpotential individuals ( $\mathrm{n} 5, \mathrm{n} 4$ and $\mathrm{nm}$ ) as one of their spatial direct parts. The boundary condition $b c 4$ is further classified as a modified interface current equation and the boundary condition $\mathrm{bcm}$ is subclassified as a standard interface current equation, both of these being material relations.

\subsection{Efficient data centric architecture}

Input data and simulation state are represented by a data model which is mapped to EMMO. This ensures variables are consistent with the physical equations, materials relations and boundary conditions. The data-models are represented as entities with a set of properties. Each properties can have a data representation shape, type (string, float, int, boolean etc) and a unit. In addition, the attribute can be mapped to specific observation methods in the EMMO that allows for documenting how the specific data point was obtained through direct- or indirect measurement or calculation. For example, the electric potential can be described as a 2D scalar field with the shape corresponding to the grid, with the unit Volt. The semantic interoperability platform SOFT [1] allows for instantiating the data model and manages data 


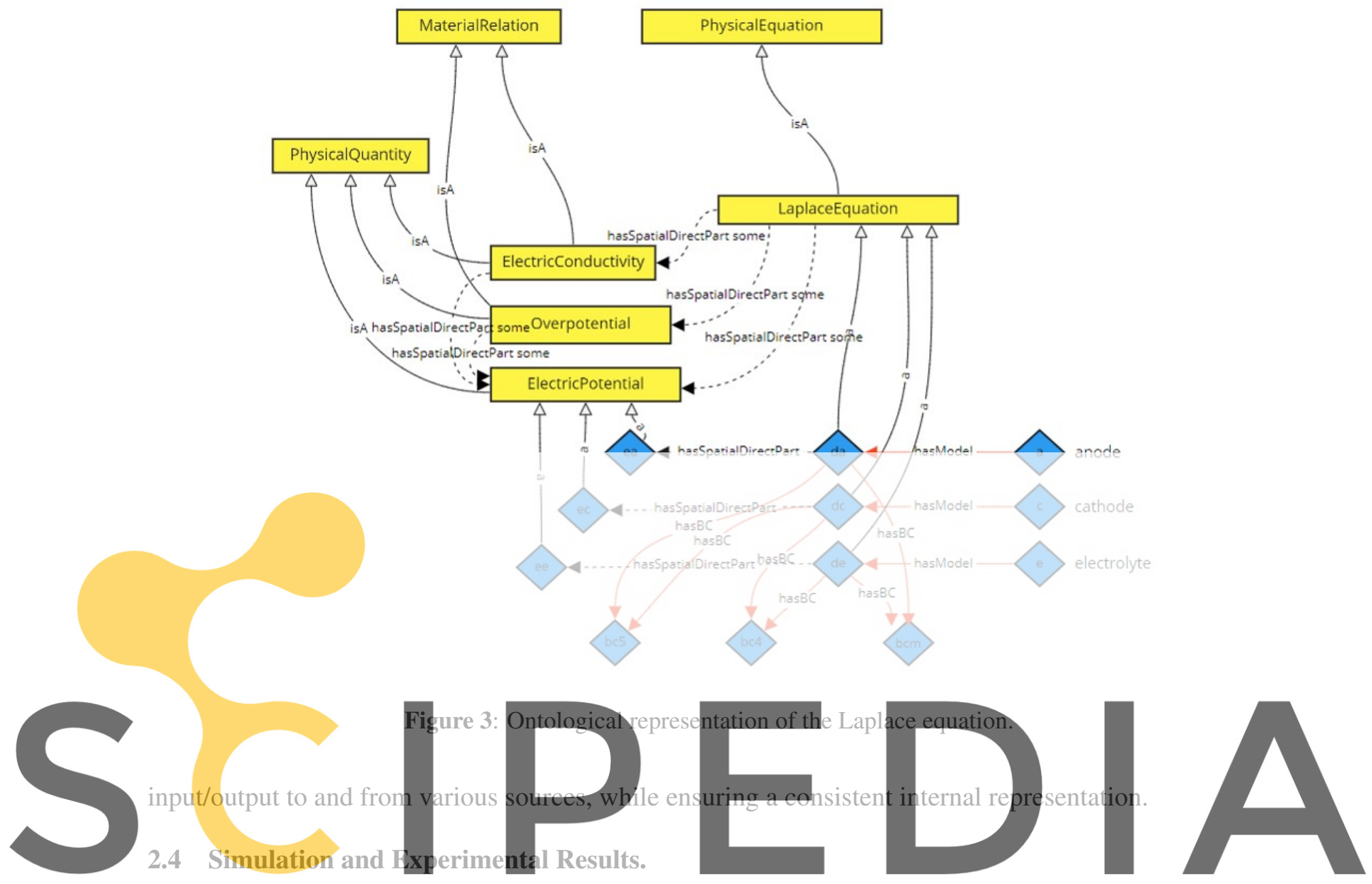

Potentiodynamic polarization measurements of the used samples reveal the electro- kinetic parameters

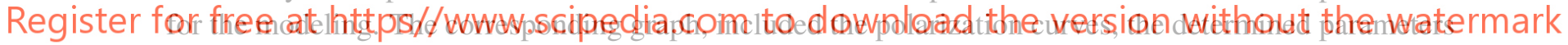
of the uncoupled bare metal samples measurements and fitted theoretical curves were also presented in [20]. In case of copper and aluminum they match the investigations of Clark et.al [16].

The fits according Murer [14] describe the kinetics over a wide range. Tafel approximation doesn't fulfill this. In this case the current $i_{d}$ limits the cathodic kinetics mainly due to (e.g. $4 e^{-}$on $\mathrm{Cu}$ ) oxygen reduction. Observations and the fitted values are in agreement with previous studies on $\mathrm{Cu}$ [17], [18] or on $\mathrm{Al}$ [19]. For very high polarization the so called hump phenomena occurs, which has not considered in the current study. Since the electrical conductivity is a very important parameter, it has been measured before and after the galvanic tests. Table 3 shows no significant variation leading to assume a constant $(55 / 1.9[\mathrm{mS} / \mathrm{cm}])$ in the simulation.

The modelling results are demonstrated in Figures 5 - 6 . The computation itself needs the direct coupling of the mentioned equations. Time steps, reinitalization, the mesh size and the interface width have to be set up very careful. For an optimized computation the following results occur. As shown in Figure 5 the electrolyte potential distribution varies mainly due to the low conductivity. Electrolyte potential (color), current flow lines (arrows) and surface deformation (red line corresponds to $\phi=0.5$ ) on the systems 


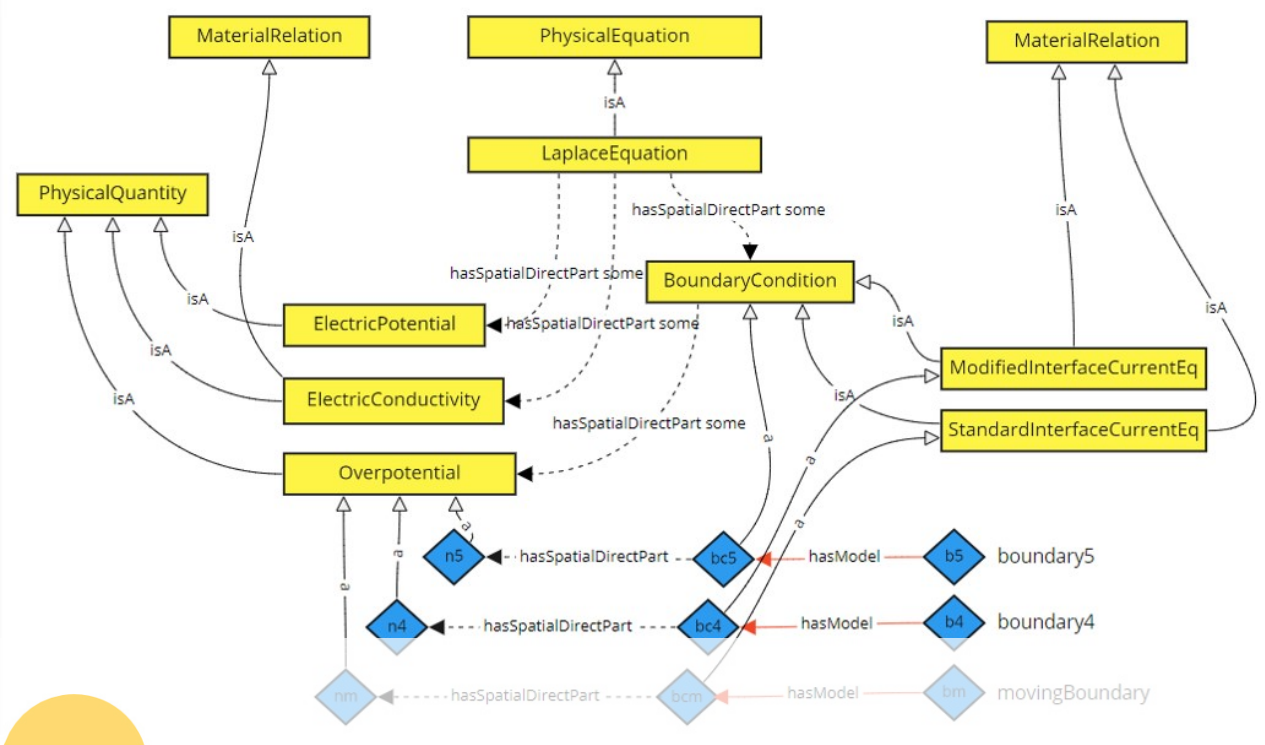

Figure 4: Ontological representation of the boundary conditions.

Table 3: Conductivities of the solutions before (first corresponding number) and after (second corresponding number) galvanic couple experiments
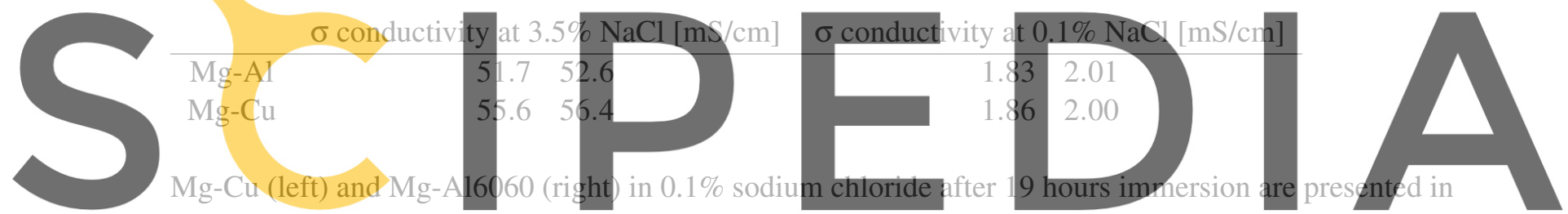

the figure. The current flow to the cathode indicates the Mg dissolution.

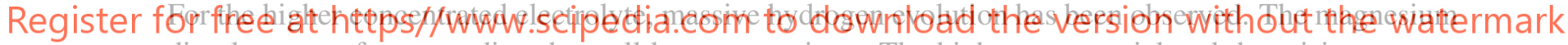

dissolves very fast according the well known reactions. The high overpotential and the arising current density of $\sim 60 \mathrm{~A} / \mathrm{cm}^{2}$ for $\mathrm{Al}$ and of $\sim 270 \mathrm{~A} / \mathrm{cm}^{2}$ for $\mathrm{Cu}$ lead to a rapid attack (see figure 6). On the figure the electrolyte potential (color), current flow lines (arrows) and surface deformation (red line - $\phi=0.5$ ) on both systems in $3.5 \%$ sodium chloride after 19 hours immersion being compared to experimental results (etched by chromic acid) are presented.

Contrary to low electrolyte conductivities the potential reached an equilibrium value as predicted by the Mixed potential theory. For copper a value of $-1.41 \mathrm{~V}$ and for aluminum of $-1.63 \mathrm{~V}$ has been determined. The comparison with the polarization curves presented in [20] provides an adequate agreement. The surface tracking by applying Faraday's law works fine. Hence, there are still certain variations. They arise from chemical conversion during immersion and massive changing conditions. The hydrogen evolution and the $\mathrm{Mg}(\mathrm{OH})_{2}$ formation strongly influence the process. Modeling of electrode surface kinetics still needs some improvements. Especially the Butler Volmer approach is just a very rough approximation, failing for high overpotentials. The equations of Murer show this possibilities, but also the limitations. 

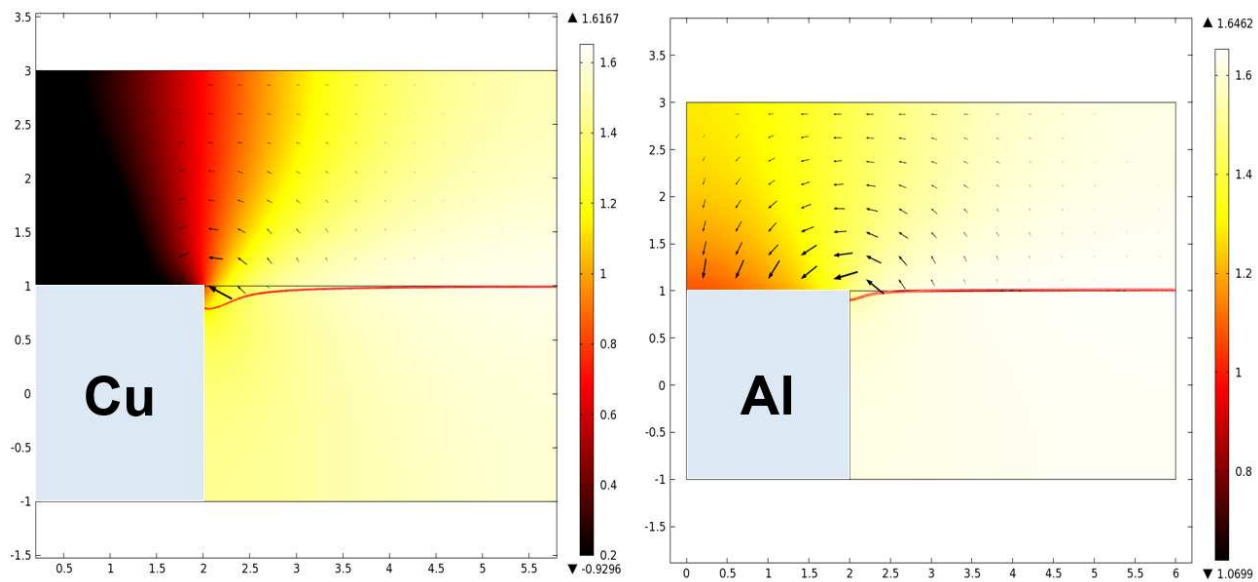

Figure 5: Modelling results for the systems $\mathbf{M g}-\mathrm{Cu}$ (left) and $\mathbf{M g}$-Al6060 (right) in $\mathbf{0 . 1 \%}$ sodium chloride after 19 hours immersion [20].

\section{Discussion}

Corrosion is a very challenging problem that involves many different fields of chemistry and physics. Particularly in this contribution authors have considered the galvanic corrosion of multi materials structures as reasonable example case. It is relevant for a number of applications for advanced constructions,

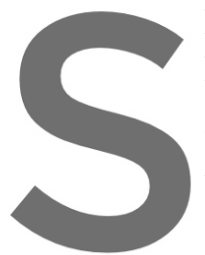
lightweight structures, logical representation is

Of course the shown numentis visualizing the transforma adequate accuracy,
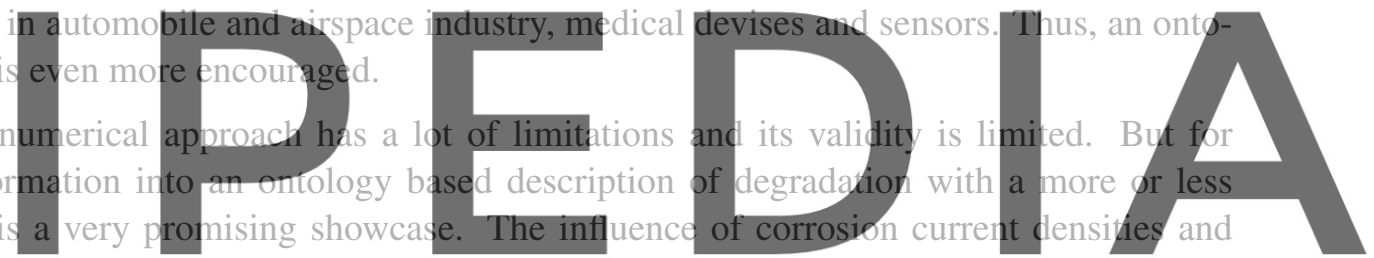

other material parameters on the process can be studied and the results computed can be utilized for

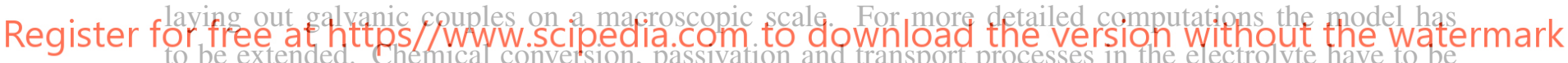
considered, which requires further modelling activities.

From a mathematical point of view, this leads to a set of non linear equations and a huge amount of degrees of freedom in FEM computations. As a secondary effect of the used Level-set method the tuning of the reinitialization parameter $\gamma_{L}$ is very difficult and should be considered and discussed separately. Thus, it is excluded from the ontological viewpoint. The next issue would be the step by step extension of the model by including migration, and chemical reaction kinetic, which is another story as well and out of scope of this work.

EMMO combined with a semantic framework will greatly simplify the integration of the needed range of models and data sources for correctly model corrosion phenomena. The domain specific ontologies presented in section 2.2 and illustrated in Figures 2 and 3 provide the input for the digitisation of galvanic corrosion phenomena. The introduced framework is semantically founded on the first outline of an EMMO-based galvanic corrosion ontology, describing the interrelations between real world objects, quantities and equations for the simple corrosion process. 

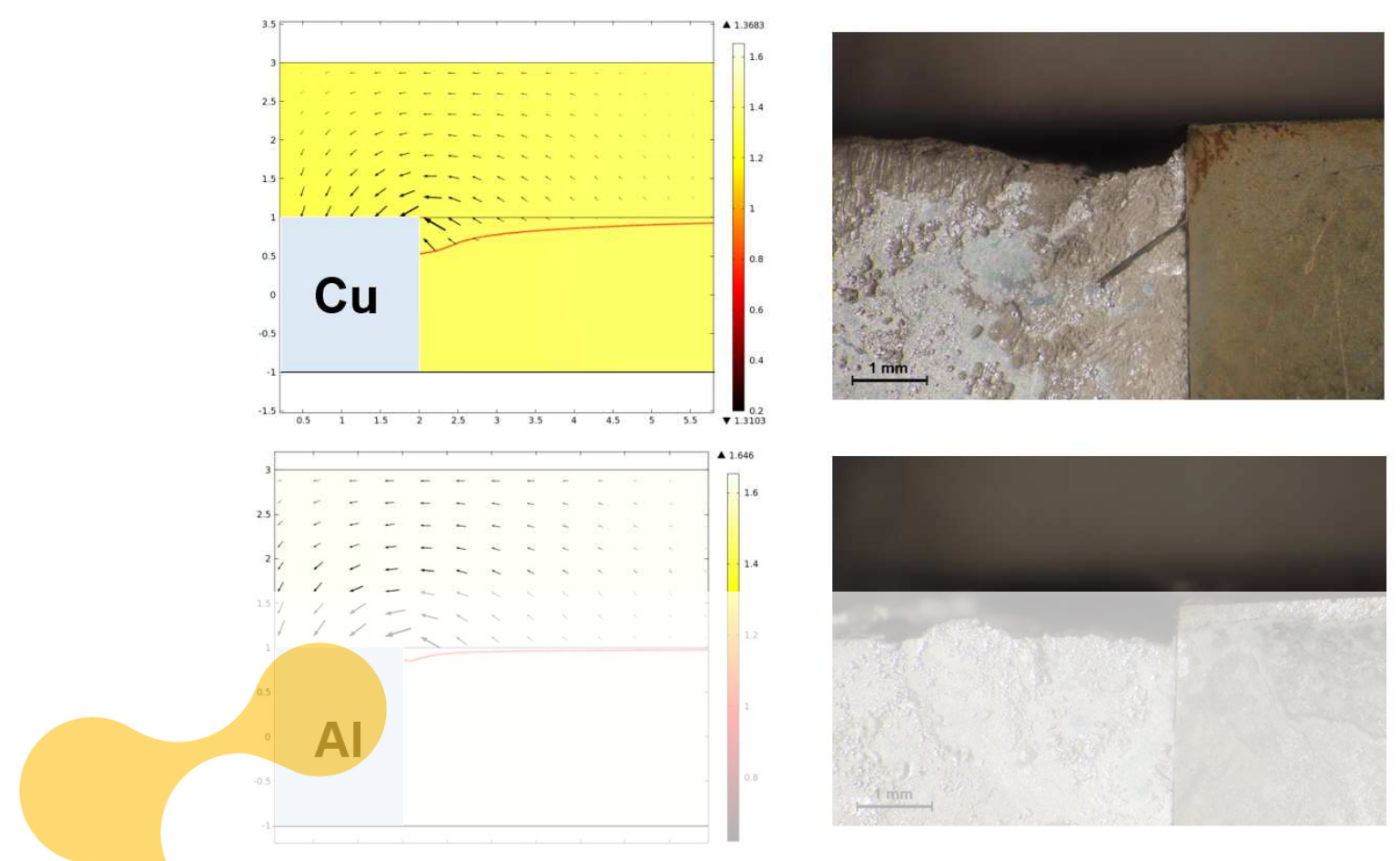

Figure 6: Modelling results for the systems $\mathrm{Mg}-\mathrm{Cu}$ and $\mathrm{Mg}$-Al6060 in 3.5\% sodium chloride after 19 hours immersion [20].

The approach indicated in rosion processes by develo important for the future de
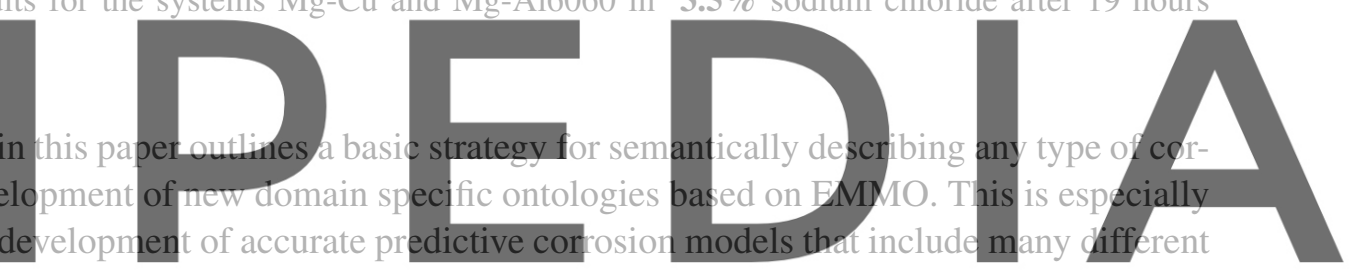

data from different scales and sources. Such models would require a sound semantic basis together with

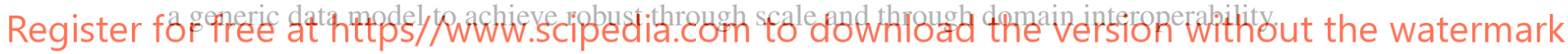

\section{Conclusions}

The work shows the development of an ontological representation of a bimetallic galvanic corrosion problem at continuum level. The physio-chemical modelling of the problem has been transferred and ontologized excluding the interface tracking method. The final achievement's are:

- the EMMO has been used for ontological representation of bimetallic galvanic corrosion

- the general description of simulation of corrosion of multi materials structures is ready for application on similar computational problems

- and progress in the machine understandable way for the data exchange respective interoperability with respect to the high level of complexity and scaling has been achieved.

The approach might build the nuclei for future developments in computational corrosion engineering.

\section{Acknowledgment}

The authors acknowledge the following European Union's research and innovation projects for the finan- 
cial support:

- OntoTrans - Ontology Driven Open Translation Environment, H2020-NMBP-10-2019, Grant agreement 862136;

- MarketPlace - Materials Modelling Marketplace for Increased Industrial Innovation, H2020-NMBP25-2017, Grant agreement 760173;

- LORCENIS - Long Lasting Reinforced Concrete for Energy infrastructure under Severe Operating Conditions, H2020-NMP-19-2015, Grant agreement 685445.

\section{REFERENCES}

[1] Mir Z.M., Friis J., Hagelien T.F., Svenum I.H., Ringdalen I., Konchakova N., Zheludkevich M.L., and Hoeche D., Interoperability architecture for bridging computational tools: Application to steel corrosion in concrete, Modelling and Simulation in Materials Science and Engineering, (2020), 28:025003.

[2] Angst U.M., Challenges and opportunities in corrosion of steel in concrete, Materials and Structures, (2018), 51:4.

[3] CEN-CWA 17284 https://www.cen.eu/news/workshops/Pages/WS-2017-012.aspx, (2018)

[4] Zander, Hoeche D., Deconinck J., Hack T., Corrosion and its context to Service-Life, Handbook of Software Solutions for ICME, (2016).

[5] Deshpande, K., Validated numerical modelling of galvanic corrosion for couples: Magnesium alloy (AE44)-mild steel and AE44-aluminium alloy (AA6063) in brine solution, Corrosion Science, (2010), 52(10): 3514-3522.

[6] Deshpande, K., Experimental investigation of galvanic corrosion: Comparison between svet and immersion techniques, Corrosion Science, (2010), 52(9): 2819-2826.

[7] Deshpande, K., Numerical modeling of micro-galvanic corrosion, Electrochimica Acta, (2011),56(4): 1737-1745.

[8] Murer N. et al., Numerical modelling of the galvanic coupling in aluminium alloys: A discussion on the application of local probe techniques, Corrosion Science, (2010), 52(1): 130-139.

[9] Jia, J. X. et al., Simulation of galvanic corrosion of magnesium coupled to a steel fastener in nacl solution, Materials and Corrosion, (2005), 56 (7) 468-474.

[10] Jia J. X., Song G. and Atrens A., Experimental measurement and computer simulation of galvanic corrosion of magnesium coupled to steel,Advanced Engineering Materials, (2007), 9 65-74.

[11] Lacroix L. et al., Localized approach to galvanic coupling in an aluminum-magnesium system, Journal of The Electrochemical Society, (2009), 156 C259-C265.

[12] Ghedini E., Goldbeck G., Friis J., Hashibon A. and Schmitz G.J. European Materials \& Modelling Ontology, https://github.com/emmo-repo/EMMO.

[13] Osher, S. and Fedkiw R. P., Level set methods and dynamic implicit surfaces, vol. 153, Springer Verlag, (2003). 
[14] Murer, N., Missert N. and Buchheit R., Towards the modeling of microgalvanic corrosion in aluminum alloys: The choice of boundary conditions, COMSOL Users Conference, (2008).

[15] Olsson, E., Kreiss, G. and Zahedi S., A conservative level set method for two phase flow ii, vol. 225, Elsevier, (2007).

[16] Clark W. J. et al.,A galvanic corrosion approach to investigating chromate effects on aluminum alloy 2024-t3, Journal of The Electrochemical Society, (2002), 149(5) B179-B185.

[17] Balakrishnan, K. and Venkatesan, V. K., Cathodic reduction of oxygen on copper and brass, Electrochimica Acta, (1979), 24 131-138.

[18] Lu, Y. et al., Predicting size effect on diffusion-limited current density of oxygen reduction by copper wire, Chinese Journal of Oceanology and Limnology, (2011), 29 75-79.

[19] Cheng, Y. L. et al., A study of the corrosion of aluminum alloy 2024-t3 under thin electrolyte layers, Corrosion Science,(2004), 46(7): 1649-1667.

[20] Hoeche, D., Isakovic, J. Level-Set modelling of galvanic corrosion of Magnesium, 2012, Proceedings of Mg2012 Conference, Vancouver, Canada. 